Disclosure of Interests: Md Yuzaiful Md Yusof: None declared, James Robinson: None declared, Yasser El-Sherbiny: None declared, Andy C Rawstron: None declared, Paul Emery Grant/research support from: Pfizer, MSD, AbbVie, Bristol-Myers Squibb, Roche, Consultant for: Pfizer, MSD, AbbVie, Bristol-Myers Squibb, UCB, Roche, Novartis, Gilead,Samsung, Sandoz and Lilly, Ann Morgan: None declared, Edward Vital Grant/ research support from: He has received honoraria and research grant support from Roche, GSK and AstraZeneca.

DOI: 10.1136/annrheumdis-2019-eular.6919

\section{SAT0010 FUNCTIONAL BIOMARKER DEVELOPMENT FOR CELL- BASED THERAPY IN RHEUMATOID ARTHRITIS}

Maya Breitman ${ }^{1}$, Tracey Bonfield ${ }^{2}$, Susan Lasalvia ${ }^{1}$, Hillard Lazarus ${ }^{3}$, Arnold Caplan ${ }^{4}$, Nora Singer ${ }^{1} .{ }^{1}$ MetroHealth System at Case Western Reserve University, Medicine division of rheumatology, Cleveland, United States of America; ${ }^{2}$ Case Western Reserve University School of Medicine, Cleveland, United States of America; ${ }^{3}$ Case Western Reserve University School of Medicine, University Hospitals Cleveland Medical Center, Cleveland, United States of America; ${ }^{4}$ Case Western Reserve University School of Medicine, Skeletal Research Center, Cleveland, United States of America

Background: Rheumatoid Arthritis (RA) is an autoimmune disease in which early treatment prevents joint damage. Autoantibodies such as rheumatoid factors (RF) and anti-citrullinated peptide antibodies (APCA) define a subset of RA patients at the highest risk for damage. Reduction of RA disease activity is associated with improvement in function(s) of regulatory $\mathrm{T}$ cells (Treg) and attenuated responses of pro-inflammatory $\mathrm{T}$ effector cells (Teff). Human Mesenchymal Stem Cells (hMSC) isolated from bone marrow and culture-expanded have strong immunomodulatory properties; we hypothesize that therapeutic use of hMSCs may skew the immune system to resemble its pre-RA state. Lack of available biologic correlates measured early-on that indicate response to treatment limits efficacy assessment of cell-based therapy. Use of clinical response criterion requires large numbers of patients and may be impractical in early stage clinical trials. This work was performed in anticipation of conducting a Phase I safety trial of cell-based therapy in early RA.

Objectives: We set out to develop functional assay(s) as biomarkers of response that would indicate early-on that hMSC were or were not modulating immune function in a manner that could potentially predict potency and efficacy in RA patients.

Methods: Sero-positive female patients with active early RA (RAPID 3 avg 5.7/10 (high severity)) and healthy donor age and sex-matched controls were enrolled. Second passage bone marrow hMSCs were obtained from healthy donors $<30$ y.o. (Case Comprehensive Cancer Center Hematopoietic Biorepository and Cellular Therapy Core). Peripheral blood CD4+ $T$ cells were stimulated by cross-linking CD2/CD3/CD28 for 5 days. CD4+ T-cell proliferation rates were measured, and suppression indices calculated by comparing proliferation indices of CD4+ T-cells cultured with and without hMSC-conditioned medium.

Results: hMSC-related suppression $32.2 \pm 7.8$ (mean + - SE) percent in RA CD4+ T-cells compared to $45.7 \pm 8.9$ percent for healthy donors CD4 + T-cells. We demonstrated that soluble products from hMSCs can inhibit proliferative responses of CD4+ $T$ cells from patients with active RA though the studies were not powered to detect differences between RA and healthy donors. With limited number of early RA patients, correlation between level of MSC-related suppression and antibody status were not possible. That being said, the data trend appears promising.

Conclusion: Our ex vivo experiments demonstrated that MSCs may be suppressive in RA as well as in healthy donors. To our knowledge, this study is the first demonstration of the potential for using ex vivo suppression assays to predict response to cell-based therapy in early RA. Such assays might be performed to predict the efficacy of selected hMSCs to treat RA.

Acknowledgement: This study was supported by David and Virginia Baldwin Foundation, and by the Clinical and Translational Science Collaborative of Cleveland, 4UL1TR0002548 from the National Center for Advancing Translational Sciences (NCATS) component of the National Institutes of Health and NIH roadmap for Medical Research. Its contents are solely the responsibility of the authors and do not necessarily represent the official views of the $\mathrm{NIH}$.

Disclosure of Interests: None declared

DOI: 10.1136/annrheumdis-2019-eular.4998

\section{SAT0011 \\ TRANSCRIPTOMICS UNVEILS UNIQUE BIOLOGICAL PROFILE OF TERTIARY LYMPHOID STRUCTURES GERMINAL CENTERS}

Serena Colafrancesco ${ }^{1}$, Elena Pipi $^{2}$, Saba Nayar ${ }^{2}$, Joana Campos ${ }^{2}$,

Valentina lannizzotto ${ }^{2}$, Francesca Arienzo ${ }^{1}$, Roberta Priori ${ }^{1}$, Guido Valesini ${ }^{1}$,

Benjamin Fisher ${ }^{2}$, Francesca Barone ${ }^{2} .{ }^{1}$ Sapienza University of Rome, Rome, Italy;

${ }^{2}$ University of Birmingham, Birmingham, United Kingdom

Background: The development of the $B$ cell repertoire is regulated by the process of affinity maturation occurring within the inner part of the $B$ cell follicles [germinal centers (GCs)] within secondary lymphoid organs (SLOs). In autoimmunity, this process might occur within aberrant aggregates of lymphocytes in target organs, such as the ectopic lymphoid structures (ELS) found in the salivary glands of patients with Sjogren's Syndrome (SS) (1). The phonotypical and functional features supporting ELS which may sustain the development of autoimmune diseases have not been identified. Moreover, functional proof that ELS contribute to the development of autoimmunity independently from SLOs has not been provided.

Objectives: To characterise the transcriptome profile of human ELS isolated from SS salivary glands in comparison with SLOs.

Methods: Frozen minor salivary gland biopsies were obtained from SS patients and selected for presence of GCs+ ELS. Human tonsils were obtained by volunteers undergoing tonsillectomy for clinical need and used as SLO control. In order to detect GCs, all samples were stained for CD21, bcl6, CD20 and CD3. Sequential sections, were stained by Cresyl Violet and GCs (CD21+ infiltrates) from both salivary glands and tonsil were selectively microdissected (Laser Capture Microdissection). Salivary gland small infiltrates and large CD21-infiltrates were microdissected too. RNA was isolated and transcribed. RNA sequencing studies were performed using ClonTech SMARTseq v4 kit. Changes and differences in the expression of specific genes of interest were confirmed with targeted PCR studies.

Results: Transcriptomics analyses revealed that GCs from ELS and SLO exhibit markedly different gene expression profiles. Of note, sequencing unveiled that GCs from ELS are characterized by an aberrant cell-proliferation profile with downregulation of BCL6 and AID, the enzymes responsible for $B$ cell affinity maturation (both $p<0.0001$ ). Also in $E L S$, progressive upregulation of CD21 transcript mirrored increases in infiltrate size and organization (being lowest in small infiltrates and progressively higher in large CD21- infiltrates and CD21+ infiltrates, $\mathrm{p}=0.002$ ). Similar data were obtained for AID, whose expression was significantly higher in large CD21+ infiltrates compared to small infiltrates $(\mathrm{p}=0.0006)$. A cytokine signature was identified in ELS GCs based on the expression of TNF, INFY, BAFF, APRIL, CXCL12, CXCL13, FAS, and FASL, all of which were upregulated compared to GCs from tonsils.

Conclusion: Our studies reveal that GCs forming in ELS exhibit marked transcriptional differences from classic GCs observed in SLOs. Main features characterizing ELS include lower levels of Bcl6 and AID, aberrant apoptosis, and a pathogenic inflammatory cytokine signature. Despite similarities in the anatomical organization and appearance of GCs in ELS and SLO, critical transcriptional differences emerge, which are likely functionally implicated in impaired regulation of the B cell cycle and survival of autoreactive B cell clones, ultimately leading to the development of autoimmune disease.

\section{REFERENCE}

[1] Barone F. et al. PNAS 2015.

Disclosure of Interests: Serena Colafrancesco: None declared, Elena Pipi: None declared, Saba Nayar: None declared, Joana Campos: None declared, Valentina lannizzotto: None declared, Francesca Arienzo: None declared, Roberta Priori: None declared, Guido Valesini: None declared, Benjamin Fisher: None declared, Francesca Barone Grant/research support from: GlaxoSmithKline, Roche, UCB Pharma, Actelion, ONO Pharmaceutical, Consultant for: GlaxoSmithKline, Roche, Actelion, ONO Pharmaceutical DOI: 10.1136/annrheumdis-2019-eular.6993 